\title{
Vocal impact on quality of life of elderly female subjects
}

Henrique Olival Costa ${ }^{1}$, Cristiane Matias ${ }^{2}$
Key words: voice, age, senior, quality, life.

\section{Summary}

\begin{abstract}
A
lthough there are several investigations focusing the physiology and anatomy of voice and the senior's larynx, little has been produced to support the knowledge of the impact of vocal conditions on quality of life of this portion of the population. Aim: To verify the impact of voice on quality of the life of elderly women, using the questionnaires Short-Form Health Survey - SF36 and Voice Handicap Index (VHI). Study Design: Prospective transversal cohort study. Method and Material: Fifty senior women participated in this research, with ages between 60 and 87 years and mean age of 70.8 years old, randomly recruited. The participants of the study were submitted to two questionnaires: SF36 and VHI. The answers of both questionnaires were compared by Kruskall-Wallis test, verifying if there were significant differences among the variables. The test of Spearman was used to evaluate if there was correlation among the results of the variables of VHI and the results obtained in the parameter of SF36 for life quality. Results: We obtained values considered statistically significant in the correlations among physical domain of VHI and physical operation, physical pain and physical role in life of SF36. Conclusion: There was a significant and positive correlation among the results obtained in the parameters physical operation, vitality, general health, mental health, corporal pain and physical role in life of SF36. There were statistically significant and negative correlations among the total results obtained in SF36 and VHI.
\end{abstract}




\section{INTRODUCTION}

Getting old is an expected phase in people's life. However, those that make it, end up suffering significant losses, such as changes to their social role, loss of relatives and friends, separation from occupational activities, and the onset of chronic diseases ${ }^{1-3}$.

Aging of the population has different characteristics depending on life conditions of each country and region. In general, the necessary conditions to reach a long life have improved all over the world, resulting in increased elderly population.

The growing number of elderly people make us wonder about the needs of this population, how it deals with daily situations, and specially, what is the psychosocial impact of aging in the routine and quality of life of elderly people ${ }^{3-10}$

The term quality of life is frequently used in the scientific context. The attention of governmental agencies to the elderly and the progression of medicine have contributed to it; thus, the elderly population has better healthcare conditions. However, this input does not necessarily mean that quality of life will also improve ${ }^{12-14}$.

One of the health conditions that may cause repercussions in quality of life is vocal conditions ${ }^{6-8}$.

Voice is a preponderant factor for communication and it reveals physical and psychological characteristics of the subject. In senescence, vocal changes may negatively interfere in the relation of subjects and in the social adjustment of elderly people ${ }^{2,6-10}$.

We found literature reports about the specific characteristics of vocal physiology and anatomy of the elderly, but little has been said about the concern to learn about the impact of vocal conditions on quality of life in this part of the population ${ }^{2,3,6,8-811}$.

In the present study, we decided to check the impact of voice on quality of life of elderly women using the Questionnaire Short-Form Health Survey - SF36 (15), adapted to Portuguese, to measure quality of life and the questionnaire Voice Handicap Index - VHI to describe the impact of voice on emotional, physical and social life of people ${ }^{16}$.

\section{MATERIAL AND METHOD}

Fifty elderly women participated in the present study; they were aged 60 to 87 years, mean age of 70.8 years, resident in a city of 200,000 inhabitants in the rural area of the state of Sao Paulo, which were randomly recruited in the streets of the city. The inclusion factors were age equal or greater than 60 years, good mental and physical health status, non-smoker, no history of vocal or neurological disorders and chronic respiratory pathway diseases.

The sample comprised fifty consecutive volunteers that met the inclusion and exclusion criteria.
All subjects signed the informed consent term approved by the Research Ethics Committee, Pontifical Catholic University of Sao Paulo.

Participants were submitted to two questionnaires that they were supposed to read and answer by themselves in writing within one hour. All relevant questions concerning the questionnaires were answered by one of the authors.

Each questionnaire has been regularly used all over the world and data collection and checking methodologies have been dully validated. We assessed the following parameters: SF36 assessed general health, mental health, vitality, body pain, physical functioning, social functioning, emotional role in life, and physical role in life. Using VHI, we observed physical, emotional and functional aspects of voice.

SF36 is a questionnaire created by Ware (1993), and it was extensively referred and approved by many areas of Human Health, and it can be applied to anyone over the age of 14 years, and consists of 8 subcategories in the concept of health: physical functioning, social functioning, body pain, vitality, emotional and social roles in life, general and mental health status. All subcategories are assessed by different questions in which the subject has to choose between different answers offered by the questionnaire that will define a final numeric score (Table 1).

VHI was proposed by Jacobson, Grywaslki, Silbergleit, Benninger and Newman (1997) and describes the impact of voice and its affections to people's lives. The instrument is divided into three subcategories: physical, emotional and

Table 1. Items assessed by different categories of questionnaire SF36.

\begin{tabular}{ll}
\hline Items & Categories \\
\hline Vigorous activities & Physical functioning \\
Moderate activities & \\
Elevate, carry vegetables & \\
Climb up the stairs & \\
Climb up one step & \\
Bend down, kneel down & \\
Walk 1 km & \\
Walk many blocks & \\
Walk one block & Physical Role \\
Take a shower, get dressed & \\
Reduces time & \\
Manages less & \\
Limited to some types & Body pain \\
Has difficulties & \\
Pain severity & General health status \\
Interfered by pain & \\
Appropriate general conditions & \\
Frequently sick & \\
Just like before & \\
Getting worse & Excellent health
\end{tabular}


functional impact. All categories have ten questions to be answered and the final count depends on the answers chosen from a list of words: never, almost never, sometimes, almost always, always.

Thus, we managed to compare what the person presented as quality of life (SF36) and vocal conditions, considering the capabilities everyone had to perform tasks that depended on their voices.

We collected responses from both questionnaires and summed up the results to reach the total final score for both and the results were compared by non-parametric KruskallWallis test, to check whether there were significant differences between the variables physical, emotional and social impact of VHI questionnaire and all subcategories of SF36. Non-parametric Spearman test was used to assess whether there was correlation between results of physical, social and emotional impact of VHI and results obtained in parameter SF36 of quality of life.

\section{RESULTS}

Table 2 shows the counts obtained for each analyzed variable using SF36.

Table 3 shows counts for variables analyzed by VHI.

Statistical analysis of VHI consistency of results is shown in Table 4.

Statistical analysis of SF36 consistency of results is shown in Table 5.

The statistical result of the comparative analysis between SF36 and VHI is shown in Table 6.

\section{DISCUSSION}

There are few studies relative to the impact of vocal condition of the elderly on quality of life. Trying to better understand this aspect, we used an instrument to measure quality of life as an expression of general health status (SF36) and an instrument to describe the psychosocial and functional impact of voice on subjects' lives (VHI). SF36 is considered an appropriate scale to measure quality of life, as suggested by Cella \& Tulsky (1990), because it assesses more than one aspect related with quality of life and measures daily activities. Lyons et al. (1994) stated that SF36 is an instrument that can be used to interview elderly people with different mental status ${ }^{17-20}$. The instrument used to measure vocal impairment (VHI), according to Jacobson et al. (1997), is designed to measure the effects of social and psychological damage of vocal affections. This opinion is shared by other authors such as Rosen \& Murry (2000) and Courey et al.(2000).

As to VHI questionnaire, $80 \%$ of the subjects presented total count between 0 and 12 points, out of a total of 120 points (Table 2). These data indicate that most of the subjects presented low level of vocal impairment, in other words, they considered their voices appropriate to their daily activities. This finding is similar to that of Rosen \& Murry (2000) when they studied the use of VHI in a group of singers and non-singers.

The responses of participants to many different subcategories of VHI were submitted to statistical analysis using Kruskall-Wallis test and there were no statistically significant differences between them $(\mathrm{p}=0.48$ physical and emotional; $p=0.52$ physical and functional; $p=0.62$ emotional and functional) (Table 4), indicating that responses given to each parameter presented similar behavior. Statistical analysis also demonstrated strong correlation between subcategories, meaning that functional, physical and emotional impairment progressed similarly (Table 2).

As to domains of questionnaire SF36, we obtained high values for means of each domain (Table 1). Each domain of SF36 questionnaire allows scores from 0 to 100. The higher the score, the better the health condition. To learn whether there was correlation between questionnaire variables, Spearman statistical correlation test was applied and did not show significant differences between them.

Spearman correlation test was applied and we established correlation between each one of the assessed parameters with SF36 questionnaire. We found strong positive correlation among the variables. This fact suggests that body pain, general health conditions and well-being feelings, depression, anxiety, and emotional control make the elderly people stop to do things or to have difficulty to finish the work because they feel that their health status limits their professional and social activities.

In order to learn whether there was any correlation between questionnaires SF36 and VHI, we applied Spearman correlation test again but this time among variables from each of the forms. We reached statistically significant values in correlations between physical domain of VHI and physical functioning, body pain and physical role in life of SF36 questionnaire.

The correlation between physical domain and physical functioning $(\mathrm{p}=0.020$ and $\mathrm{r}=-0.325)$ may mean that the change in vocal quality, difficulty to talk and vocal instability, which are present in aging, may cause limitations in the conduction of daily physical tasks such as: going shopping, walking, taking a shower, and practicing sports. We observed that even though they did not have use of voice in physical activities assessed by the physical functioning scale, these two aspects - physical domain and functioning physical aspect - walked hand-in-hand.

The results that presented high physical impairment, without interference in specific activities, could have indicated that subjects were well adapted, regardless of vocal characteristics present in aging. However, abnormalities to vocal quality, effort when speaking, instability and vocal fatigue may be some of the factors that lead the elderly population to seek for healthcare services. Based on it, we 
Table 2. Numeric results obtained for each variable of SF36.

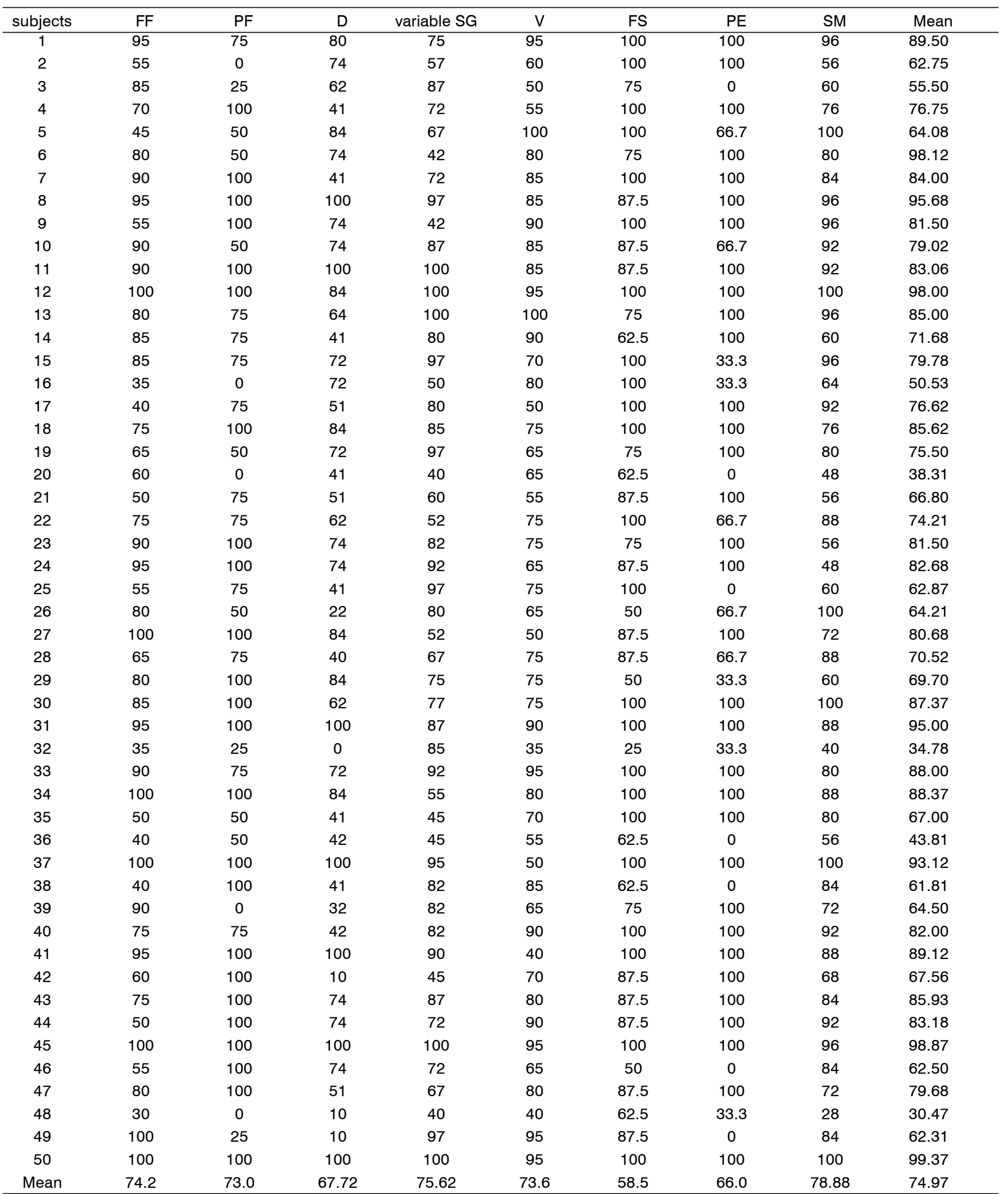

FF - Physical functioning; PF - physical role in life; D - body pain; SG - general health; V - vitality; FS - social functioning; PE - emotional role in life; SM - mental health. 
Table 3. Numeric results obtained for each variable of VHI.

\begin{tabular}{|c|c|c|c|c|}
\hline \multirow[t]{2}{*}{ subjects } & \multicolumn{3}{|c|}{ variable } & \multirow[b]{2}{*}{ Total } \\
\hline & Physical & Emotional & Functional & \\
\hline 2 & 9 & 8 & 7 & 24 \\
\hline 4 & 0 & 4 & 2 & 6 \\
\hline 5 & 0 & 0 & 0 & 0 \\
\hline 6 & 0 & 0 & 0 & 0 \\
\hline 9 & 2 & 4 & 1 & 7 \\
\hline 10 & 0 & 0 & 0 & 0 \\
\hline 11 & 0 & 3 & 1 & 4 \\
\hline 12 & 4 & 2 & 2 & 8 \\
\hline 13 & 0 & 2 & 3 & 5 \\
\hline 14 & 6 & 4 & 2 & 12 \\
\hline 20 & 3 & 2 & 3 & 8 \\
\hline 21 & 2 & 2 & 2 & 6 \\
\hline 22 & 24 & 21 & 13 & 58 \\
\hline 23 & 0 & 0 & 2 & 2 \\
\hline 24 & 0 & 0 & 0 & 0 \\
\hline 25 & 2 & 6 & 0 & 8 \\
\hline 26 & 8 & 0 & 2 & 10 \\
\hline 27 & 0 & 0 & 0 & 0 \\
\hline 28 & 6 & 15 & 14 & 35 \\
\hline 29 & 11 & 8 & 0 & 19 \\
\hline 30 & 0 & 3 & 2 & 5 \\
\hline 31 & 0 & 0 & 0 & 0 \\
\hline 40 & 0 & 0 & 1 & 1 \\
\hline 41 & 0 & 0 & 0 & 0 \\
\hline 42 & 0 & 0 & 0 & 0 \\
\hline 43 & 0 & 0 & 0 & 0 \\
\hline 44 & 2 & 0 & 0 & 2 \\
\hline 45 & 6 & 0 & 0 & 6 \\
\hline 46 & 2 & 0 & 0 & 2 \\
\hline 47 & 0 & 0 & 0 & 0 \\
\hline 48 & 0 & 0 & 7 & 7 \\
\hline 49 & 0 & 0 & 0 & 0 \\
\hline 50 & 0 & 0 & 1 & 1 \\
\hline Mean & 3.38 & 2.32 & 2.78 & 8.48 \\
\hline
\end{tabular}

Table 4. $\mathrm{p}$ values for the comparative analysis of each $\mathrm{VHI}$ variable.

\begin{tabular}{cccc}
\hline Pair of variables & Physical vs. Emotional & Physical vs. Functional & Emotional vs. Functional \\
\hline $\mathrm{p}$ value & $0.48(\mathrm{~N} . \mathrm{S})$ & $0.52(\mathrm{~N} . \mathrm{S})$ & 0.62 (N.S) \\
\hline & & & \\
\hline
\end{tabular}


Table 5. $p$ and $r$ values for correlation of subcategories of SF36 when there were statistically significant differences (Spearman test, $p \leq 0.05)$

\begin{tabular}{lcc}
\hline Subcategory pairs & p value & $r$ value \\
Physical Functioning versus physical role in life & 0.0033 & 0.407 \\
Physical Functioning versus body pain & 0.0013 & 0.514 \\
Physical Functioning versus general health status & 0.00062 & 0.535 \\
Physical Functioning versus Vitality & 0.011 & 0.353 \\
Physical role in life versus body pain & 0.00010 & 0.520 \\
Physical Role in life versus Vitality & 0.037 & 0.295 \\
Physical Role in life versus Mental health status & 0.019 & 0.3306 \\
Body pain versus General health status & 0.016 & 0.339 \\
Body pain versus Vitality & 0.021 & 0.324 \\
Body pain versus Mental health status & 0.019 & 0.428 \\
General health status versus Vitality & 0.005 & 0.3841 \\
General health status versus Mental health status & 0.0064 & 0.3805 \\
Vitality versus Mental health status & 0.000001 & 0.625
\end{tabular}

Table 6. $p$ and $r$ values for the correlation between subcategories of SF36 and VHI categories (Spearman test, $p \leq 0.05$ )

\begin{tabular}{lccc}
\hline SubcategoriesVHI & SF36 & r values & $p$ values \\
Physical Functioning x physical & & -0.325 & 0.020 \\
Physical x physical Role in life & & -0.315 & 0.025 \\
Functional x physical Functioning & & -0.044 & 0.0010 \\
Functional Role x physical in life & & -0.453 & 0.0009 \\
Functional x Body pain & -0.35 & 0.010 \\
\hline
\end{tabular}

can deduct that when working with elderly people, therapeutic approach should focus on preventive aspects and increment of vocal efficiency. To minimize the effects of presbiphonics, specific vocal training should be addressed to vocal quality and speech and articulation dynamics. Moreover, Morrison \& Rammage (1994) and Sataloff; Rosen; Hawkshaw; Spiegel (1997) stated that the effects of aging in voice can be less evident in the presence of good physical conditions.

Once established the correlations and consistencies between the various parameters in each questionnaire, we investigated the correlation between total counts of VHI and SF36. Statistical results did not show statistically significant differences. However, the analysis of values obtained for each subject showed that only three of them had high total count of VHI. Women with extreme impairment were excluded from the analysis and covariate analysis was conducted in the rest of the group, showing strongly negative covariation $(\mathrm{x}=-48.78)$, or in other words, the higher the vocal impairment, the worse the quality of life. Spearman correlation test was also applied and we reached correlation between total count of SF36 and VHI as $r=-0.45$ and $p=$ 0.049 , showing that there was significant negative correlation between quality of life and vocal impairment, confirming the results described above.

These results are in accordance with the study by Benninger et al. (1998), conducted to assess the correlation between SF36 domains and VHI categories in patients with vocal affections, in which they detected that vocal affections had significant impact on quality of life of subjects. Similarly, the study by Ferreira et al. showed impact on quality of life and lifestyle of patients (1994).

\section{CONCLUSION}

- Physical, emotional and social parameters of VHI were coherent and correlated;

- There was statistically significant positive difference between results obtained in physical functioning, vitality, general health status, mental health, body pain and physical role in life from SF36;

- There was statistically significant and negative difference between total result obtained in SF36 and VHI. Thus, vocal conditions may significantly interfere in quality of life of women aged over 60

\section{REFERENCES}

1. Von Leden H, Alessi DM. The aging voice. In: Benninger MS, Jacobson BH, Johnson AF. Vocal arts medicine. The care and prevention of professional voice disorders. New York: Thieme; 1994 p. 269-80.

2. Sataloff RT, Rosen DC, Hawksha M, Spiegel JR. The three ages of voice: the aging adult voice. J Voice 199711 (2): 156-60. 
3. Morrison M, Rammage L. Voice disorders in the elderly. In: The management of voice disorders. San Diego, California: Singular Publishing; 1994. p.141-9.

4. Sato K, Hlrano M. Age-related changes of elastic fibers in the superficial layer of the lamina propria of vocal folds. Ann Otol Rhinol Laryngol 1997; 106: 44-7.

5. Wilcox KA, Horii Y. Age and changes in vocal jitter. J Gerontol 1980; 35(2): 194-8.

6. Woo P, Casper J, Colton R, Brewer D. Dysphonia in the aging: physiology versus disease. Laryngoscope 1992; 102: 139-44.

7. Allodi PM, Ferreira LP. A voz no envelhecer. In: Ferreira LP, Costa HO. Voz ativa - falando sobre a clínica fonoaudiológica. São Paulo: Ed. Roca; 2001. p.219-35.

8. Ramig LA, Ringel RL. Effects of physiological aging on selected acoustic characteristics of voice. J Speech Hear Res 1983; 26: 22- 30.

9. Decoster W, Debruyne F. The aging voice: changes in fundamental frequency waveform stability and spectrum. Acta Otorhinolaryngol Belg 1997; 51: 105-12.

10. Duprata C. Influência hormonal na voz. In: Costa HO, Andrada e Silva MA. Voz cantada - evolução, avaliação e terapia fonoaudiológica. São Paulo: Lovise; 1998. p.115-7.

11. Hagen P, Lyons GD, Nuss DW. Dysphonia in the elderly: diagnosis and management of age-related voice changes. Southern Med J 1996; 89(2): 204-7.

12. Cella DF, Tulsky DS. Measuring quality of life today: methodological aspects. Oncology 1990; 4(5): 29-38.
13. Miettinen OS. Quality of life from the epidemiologic perspective J Chr Dis 1987; 40(6): 641-3.

14. Palmore E, Lulkart C. Health and social factors related to life satisfaction. J Health \& Social Behav 1972; 13: 68-80.

15. Ware JE Jr. SF-36 health survey: manual \& interpretation guide. Quality metric incorporated. Lincoln Rhode Island; 1993. 293 p.

16. Jacobson BH, Grywalski C, Silbergleit A, Jacobson G, Benninger MS, Newman CW. The voice handicap index(VHI): development and validation. Am J Speech-Language Pathol 1997; 6(3): 66-70.

17. Brazier JE, Harper R, Jones NMB, O'cathain A, Thomas KJ, Ushe Wood T, Westlake L. Validating the SF-36 health survey questionnaire: new outcome measure for primary care. $\mathrm{Br}$ Med J 1992; 6846 (305): 129-202.

18. Ware JJ, Sherbourne CD. The MOS 36 item short-form health survey (SF-36). I conceptual framework and item selection. Med Care 1992; 30: 473-83.

19. Jenkinson C, Wright L, Coulter A. Criterion validity and reliability of the SF-36 in a population sample. Quality life Res 1994; 3: 7-12.

20. Lyons RA, Perry HM, Littlepage BNC. Evidence for the validity of the short-form 36 questionnaire (SF-36) in a elderly population. Age and aging 1994; 23: 182-4.

21. Rosen CA, Murry T. Voice handicap index in singers. J Voice 2000; 14 (3): 370-7.

22. Smith E, Nichols S, Lenke J, Dove H \& Hoffman H. Effects of voice disorders on patient lifestyle: preliminary results. Ncvs status and progress report 1994: 237-48. 